\title{
Diacronie
}

Studi di Storia Contemporanea

$\mathrm{N}^{\circ} 28,4 \mid 2016$

La voce del silenzio

\section{Antonia Lovecchio, Professione rivoluzionario. Per una biografia di Ruggero Grieco (1893-1926)}

Andrea Ricciardi

\section{Q OpenEdition \\ Journals}

Edizione digitale

URL: http://journals.openedition.org/diacronie/4647

ISSN: 2038-0925

Editore

Association culturelle Diacronie

Notizia bibliografica digitale

Andrea Ricciardi, « Antonia Lovecchio, Professione rivoluzionario. Per una biografia di Ruggero Grieco (1893-1926) », Diacronie [Online], № 28, 4 | 2016, documento 27, Messo online il 29 décembre 2016, consultato il 22 septembre 2020. URL : http://journals.openedition.org/diacronie/4647 


\section{RECENSIONE:}

\section{Antonia LOVECCHIO, Professione rivoluzionario. Per una biografia di Ruggero Grieco (1893-1926), Bari, Edizioni del Sud, 2013, 212 pp.}

a cura di Andrea RICCIARDI *

Il volume dimostra che, sebbene non sembri più "di moda", la storia politica è una branca della storia contemporanea ancora capace di fornire chiavi di lettura fondamentali del Novecento, considerati anche gli archivi di persone che sono stati aperti alla consultazione solo in questi ultimi anni e che, se adeguatamente esplorati, si configurano come importanti occasioni per approfondire temi e filoni di ricerca tutt'altro che esauriti.

Lovecchio, attraverso un uso intelligente delle fonti primarie e secondarie, tratteggia la prima parte della vicenda politica (e personale) di Grieco che, in realtà, non è solo la storia di un singolo militante e dirigente comunista, ma è anche un percorso esemplificativo di una stagione, chiusa dalle leggi "fascistissime" del novembre 1926, ricca (non soltanto per i membri del PCd'I) di sogni, contraddizioni, passioni, ingenuità e drammi. In quest'ottica, è inevitabile ricollegare il volume almeno alle tre monografie recentemente dedicate a Luigi Longo, Pietro Secchia ed Eugenio Curiel da Höbel, Albeltaro e Fresu che, pur spingendosi ben oltre gli anni trattati da Lovecchio, hanno affrontato come lei (con stili diversi tra di loro) la vita di un rivoluzionario "professionale" del Novecento calato nel suo tempo".

\footnotetext{
${ }^{1}$ Cfr. HÖBEL, Alexander, Luigi Longo. Una vita partigiana (1940-1945), introduzione di Aldo Agosti, Roma, Carocci, 2013; FRESU, Gianni, Eugenio Curiel. Il lungo viaggio contro il fascismo, Roma, Odradek, 2013; ALBELTARO, Marco, Le rivoluzioni non cadono dal cielo. Pietro Secchia, una vita di parte, Roma-Bari, Laterza, 2014. Nell'ultimo decennio, oltrepassando l'ambito comunista e segnalando soltanto alcuni lavori, ricordo che sono state pubblicate monografie su dirigenti politici del calibro di Riccardo Lombardi, Lelio Basso,
} 
Lovecchio racconta la prima parte della vita di Grieco in cinque capitoli, seguiti da alcune note conclusive in cui riflette sulla natura del suo meridionalismo nell'Italia repubblicana, provando a comprendere se (e in che misura) le sue battaglie condotte tra gli anni Quaranta e la scomparsa, sopravvenuta nel 1955, si possono connettere con le riflessioni giovanili e l'attività svolta fino alla definitiva affermazione del fascismoregime. A questo proposito, augurandoci che Lovecchio possa completare la biografia affrontando con altrettanta acribia gli anni successivi al 1926, sarebbe stato forse opportuno aprire qualche parentesi più ampia sul Grieco degli anni Trenta. L'autrice accenna ad alcuni nodi sia nell'introduzione, nella quale spiega il percorso di ricerca compiuto e fornisce vari chiarimenti sulle carte compulsate, sia nei capitoli. Ma sfiora soltanto le svolte politiche di fine anni Venti e metà anni Trenta, connesse con il VI e il VII Congresso del Comintern, e le conseguenti posizioni di Grieco in rapporto a questioni molto discusse in questi ultimi anni, a cominciare dal rapporto con Gramsci e senza dimenticare i profondi rivolgimenti al vertice di un PCd'I accusato di scarsa "vigilanza rivoluzionaria” da Mosca, proprio nella fase più terribile delle purghe staliniane che si riverberarono oltre i confini dell'URSS.

Ciò non inficia in alcun modo il valore di un volume che, pur dando forse poco spazio al contesto "esterno" al partito nel quale Grieco e i suoi compagni si mossero fino alla fine del $1926^{2}$, dimostra quanto sia importante tenere presente la complessità dello sviluppo storico, senza nascondere la propria sensibilità soggettiva ma, nel contempo, non cedendo alla tentazione (molto diffusa anche tra i giovani studiosi) di giudicare il passato tout court, maneggiando con eccessiva disinvoltura categorie e vicende molto ricche di sfaccettature e, per questo, da trattare sì con passione ma anche con una troppo spesso dimenticata (o sconosciuta) umiltà intellettuale.

Grieco nasce a Foggia nel 1893 e, scomparso prematuramente il padre Giuseppe, è mandato dalla madre Linda (madre di altre tre figlie) al convitto di Spoleto, per lui unica reale possibilità di proseguire gli studi. È lì che Ruggero, come egli stesso ricorderà in un secondo tempo, si confronta con i primi testi di argomento politico, a

Antonio Giolitti, Ugo La Malfa, Leo Valiani, Ernesto Rossi, Altiero Spinelli, Sandro Pertini e Alcide De Gasperi.

${ }^{2}$ Gli altri partiti antifascisti, compresi il PSI e il PSLI, rimangono sullo sfondo della narrazione. Vista l'attenzione per i singoli documenti e scritti prodotti da Grieco, talvolta ampiamente citati per coglierne le più sottili sfumature, sarebbe stato forse opportuno soffermarsi un po' di più sulle strategie degli interlocutori del comunista foggiano e del PCd'I, al fine di far emergere il contesto politico-istituzionale italiano in tutta la sua drammatica complessità, per cogliere meglio come il fascismo si affermò e quali furono le numerose inadeguatezze dei suoi oppositori. Anche di quelli più radicali, spesso incapaci di cogliere in tempo la pericolosità di Mussolini e portati a far valere le ragioni di partito rispetto alla costruzione di un fronte unico di opposizione, soprattutto dopo il delitto Matteotti e le incertezze di Vittorio Emanuele III in corrispondenza della scelta aventiniana. 
cominciare dalle opere di Mazzini, per poi prendere i primi contatti con il PSI. Grieco riesce dunque, pur tra non poche difficoltà, ad approcciarsi alla politica in un contesto particolare. Tuttavia l'istruzione politica di un autodidatta risulta inevitabilmente disordinata e priva di metodo, anche perché essa si accompagna a una chiara propensione per le discipline umanistiche (l'italiano, in particolare), che lo impegnano molto. Ruggero è un eccellente studente ma, dopo aver concluso gli studi primari, decide di iscriversi alla sezione agronomica dell'istituto tecnico, «decisione con ogni probabilità dettata dall'ambizione a trovare un impiego nella sua terra d'origine, la Capitanata, in massima parte agricola»3 3 . La passione per la letteratura, però, rimane intatta e Ruggero collabora nel 1911 con «Juventus», una rivista letteraria per ragazzi stampata a Firenze, in cui compaiono vari contributi dedicati a D’Annunzio. Le sue opere, complice un breve idillio amoroso del Vate con la madre, vengono elogiate con passione ma il nazionalismo che pervade la rivista, la quale sostiene l'impresa libica con entusiasmo, non può coinvolgere il giovane che, di lì a poco, sarebbe entrato nel PSI. Diverso, e molto critico, risulta essere il suo approccio a Marinetti e al movimento futurista, nel quale Grieco scorge un pericolo: una concezione del progresso incentrata sulla purificazione derivante dalla guerra può portare danni e squilibri profondi, anche fuori dall'Italia.

Conseguito il titolo di perito agronomo, Ruggero si ricongiunge con la madre e le due sorelle Armida e Mara (Ida era scomparsa da piccolissima per un incidente domestico). È questa la fase in cui egli inizia la militanza politica al fianco dei braccianti, iscrivendosi alla sezione di Foggia del PSI nel 1912. Alla fine dell'anno si trasferisce però a Portici, con l'intento di proseguire i suoi studi di agronomia presso la prestigiosa Scuola Superiore di Agricoltura. Ma, dopo un anno e sempre per problemi economici connessi con la scomparsa della madre a soli 51 anni (da tempo era malata di cuore), Grieco deve interrompere il corso e cercare un impiego. E' in questo frangente che avviene l'incontro con Bordiga, già allora esponente di spicco del socialismo partenopeo e fondatore, con altri giovani della sinistra intransigente, di un circolo rivoluzionario intitolato a Carlo Marx, pochi mesi prima dell'espulsione dei riformisti di Bissolati e Bonomi dal PSI. I contrasti generazionali sono un elemento centrale di questa stagione: il dualismo tra "destri" e "sinistri”, quindi, non può essere sufficiente per rappresentare al meglio il quadro di una situazione in rapida evoluzione. La lotta al parlamentarismo e al compromesso elettorale è il fulcro della linea della sinistra rivoluzionaria: Grieco collabora con «La Voce», organo del circolo "Carlo Marx”, ma

3 Cfr. LOVECCHIO, Antonia, Professione rivoluzionario. Per una biografia di Ruggero Grieco (1893-1926), Bari, Edizioni del Sud, 2013, p. 27. 
anche con «Il Socialista» di Napoli e con «Il Lavoro» di Portici, sebbene inizialmente venga accettato in questi contesti con una qualche diffidenza, derivante dalle sue precedenti passioni per Mazzini e per i repubblicani. Una questione centrale, in questa fase, è costituita dalla legittimità del ricorso alla violenza, di fronte alla repressione delle proteste popolari seguite all'inasprimento dei dazi da parte del governo. A questo tema si affianca quello delle candidature alle imminenti elezioni politiche, su cui i giovani socialisti intransigenti (guidati da Bordiga) esprimono posizioni oltranziste.

È allora che Grieco conosce Ines Gambarini, nipote del socialista rivoluzionario Mario Bianchi, successivamente approdato al fascismo. Con lei, Grieco si trasferisce a Roma in cerca di un'occupazione fissa. Non perde però i legami con il contesto partenopeo: si sposa il primo agosto 1914, Bordiga gli fa da testimone di nozze. Nonostante il suo antimilitarismo, allo scoppio della guerra Grieco deve prendere parte alle operazioni e viene arruolato nel giugno 1915 come soldato semplice. Promosso sottotenente di milizia territoriale a Codevigo, in provincia di Padova, Grieco viene raggiunto da Ines che, poche settimane prima che il marito si rechi al fronte in Friuli, dà alla luce il loro primogenito Sergio. La guerra non consente a Grieco di partecipare all'assemblea fiorentina della corrente massimalista e rivoluzionaria che, nel novembre 1917 e in corrispondenza della rivoluzione bolscevica, registra il primo incontro tra Bordiga e Gramsci. Alla fine del 1918 nasce «Il Soviet», nuovo organo della sinistra intransigente. Grieco, tornato dal fronte, riprende i contatti con i compagni napoletani ma, raggiunti a Roma moglie e figlio, non abbandona la sua passione per la letteratura scrivendo articoli di critica letteraria: uno è ospitato da «Comunismo», quindicinale fondato nel 1919 da Serrati (che entrerà nel PCd'I con i “terzini” del PSI nel 1924) grazie al sostegno economico dei bolscevichi . Ma la politica è il centro dei suo interessi, in un clima che sembra prossimo alla rivoluzione. Nel febbraio 1920, Grieco entra nella segreteria del PSI come "aggregato alla segreteria", primo e ultimo incarico in quel partito. Infatti il gruppo de «Il Soviet», nel gennaio del 1921, si pone alla testa della scissione di Livorno da cui nasce il PCd'I: Bordiga ne diviene il segretario e Grieco collabora attivamente con lui per affermare le posizioni più intransigenti, accettando tutte le condizioni poste dal Comintern a cominciare dall'ultima, proposta proprio da Bordiga: l'espulsione di tutti i compagni in disaccordo con la linea di Mosca.

Grieco si occupa inizialmente del lavoro di organizzazione e, come il resto dei vertici comunisti, mostra posizioni settarie e per molti aspetti slegate dalla realtà del paese. La rivoluzione (dopo il fallimento dell'occupazione delle fabbriche) non è alle porte, i fascisti imperversano e i comunisti, pur scegliendo di presentarsi alle elezioni in linea

4 Ibidem, p. 49. 
con le indicazioni date del Comintern, possono contare su poco meno di 300.000 consensi mentre il PSI, ancora unito (la rottura tra i massimalisti e i riformisti guidati da Turati, Treves e Matteotti avverrà all'inizio del fatidico ottobre 1922), ha ancora più di un 1.300.00o sostenitori. All'attenzione per i cattolici, a cui si lega quella per i contadini poveri, non corrisponde quella per il "fiumanesimo" né per gli Arditi del popolo. Le oscillazioni del PCd'I rispetto agli Arditi sono motivo di tensione con il Comintern che, riunito a congresso, sembra orientato a mutare la strategia precedentemente adottata rispetto alle altre forze politiche senza convincere Bordiga e $\mathrm{i}$ suoi compagni, ad eccezione di Gramsci. Lovecchio scrive:

\begin{abstract}
A fronte dell'esortazione a trasformare i partiti comunisti in formazioni di massa, il gruppo dirigente italiano perseverò nella via dell'isolamento settario, mantenendo le distanze da tutti i tentativi di resistenza dal basso posti in essere dalle formazioni militari che si venivano organizzando autonomamente nella penisola. Grieco fu tra i più convinti sostenitori di questa politica miope e fallimentare5.
\end{abstract}

Mentre il fascismo dilaga e il Comintern caldeggia la formazione di un fronte unico, pur non indicando come giungere concretamente a un'azione unitaria, Grieco porta avanti con convinzione le ragioni dell'antifusionismo, attaccando innanzitutto la socialdemocrazia e ribadendo la necessità per il PCd'I di mantenere una sorta di purezza rivoluzionaria rispetto alle diverse articolazioni del movimento operaio. Qualche concessione può riguardare il campo sindacale, anche se mancano le condizioni perché si crei una reale unità di intenti. La nascita dell'Alleanza del Lavoro (febbraio 1922) non muta il quadro: il fascismo viene rigidamente interpretato come un mero strumento della borghesia liberale, l'isolazionismo sembra la via maestra per fronteggiare un avversario le cui caratteristiche sfuggono non solo a Grieco, ma alla quasi totalità dei vertici comunisti (a cominciare da Bordiga). La rottura dell'unità del PSI rappresenta un altro terreno di confronto, non solo ai vertici del PCd'I: Togliatti mostra un'apertura al Comintern mentre Bordiga, in pieno accordo con Grieco, si rifugia in una posizione di totale chiusura ad ogni dialogo con i socialisti massimalisti che contempli la messa in discussione dell'esito di Livorno.

Dopo la Marcia su Roma e la nascita del Governo Mussolini, tuttavia, il PCUS (con Lenin, Trockij, Zinov'ev, Radek e Bucharin) ottiene dal PCd'I una "sottomissione formale" alla fusione con il PSI. Grieco (con Bordiga e Terracini) sembra piegarsi alle decisioni di Mosca, anche se Livorno ha ormai creato lacerazioni politiche e personali

\footnotetext{
5 Ibidem, p. 60.
} 
impossibili da ricomporre anche per i socialisti, a cominciare da Nenni e da Vella. Nel frattempo la repressione del dissenso da parte dei fascisti si intensifica: Bordiga e vari membri del Comitato Esecutivo del PCd'I vengono arrestati. Grieco elogia il segretario con il quale si sente in totale sintonia, anche rispetto a Gramsci. Ma lo stesso Grieco, nel marzo 1923, viene arrestato e, nel carcere romano di Regina Coeli, partecipa a un dibattito interno - mediante un carteggio clandestino - che prefigura i contrasti con il futuro gruppo dirigente di centro, guidato da Gramsci e Togliatti. Il Comintern, sempre più critico con Bordiga e la sua visione dei rapporti con i socialisti, impone un nuovo Comitato centrale ma la sconfessione del gruppo bordighiano non si traduce, per il momento, in un autentico rivolgimento degli equilibri interni al partito. Gli stessi Bordiga e Grieco si dimettono poi dal CC, mentre il primo processo ai vertici del partito si traduce in uno smacco per il fascismo, vista l'assoluzione di quasi tutti gli imputati. Proprio in questa fase, tuttavia, si pongono le basi per la rottura tra la sinistra bordighiana e il centro gramsciano. Anche secondo la successiva ricostruzione di Giorgio Amendola ${ }^{6}$, durante la detenzione Grieco opera un primo (e parziale) ripensamento critico della sua esperienza politica. Uscito dal carcere, diviene responsabile dell'Ufficio stampa e propaganda del partito mentre Bordiga, rientrato a Napoli, tende a isolarsi declinando ogni responsabilità, ma continuando a considerare il compagno un suo degno sostituto negli organi dirigenti.

Nei primi mesi del 1924, mentre Bordiga fonda «Il Prometeo» (chiuso ad agosto su decisione della centrale comunista), Grieco collabora frequentemente con «Unità», «Ordine Nuovo» e «Stato Operaio», testate organiche alla nuova direzione di centro. Subisce ancora l'ascendente del primo segretario del PCd'I ma ora, scrive Lovecchio, ne ha individuato alcuni limiti che sempre più spesso saranno attribuiti a Bordiga dai compagni: «ossia la tendenza a far derivare la pratica dalla teoria, a ragionare per schemi matematici, ad ancorare l'azione a principi immodificabili» 7 . Candidato alle elezioni (al contrario di Bordiga) ma non eletto, Grieco è ancora schierato con la sinistra ma la sua collaborazione con Gramsci cresce sulla base del comune interesse per il Mezzogiorno. L'idea che la lotta dei contadini meridionali si debba saldare con quella dei proletari industriali del Nord, pur diversamente declinata, appartiene a entrambi anche se l'atteggiamento critico di Grieco verso la posizione del Comintern, in linea con Bordiga, non muta. Tra il 1924 e l'inizio del 1926, il nuovo gruppo dirigente che ruota intorno a Gramsci si consolida. Spiega Lovecchio: «la vicenda personale e

\footnotetext{
${ }^{6}$ Il riferimento è alla prefazione di Giorgio Amendola a GRIECO, Ruggero, Scritti scelti, vol. I, Roma, Editori Riuniti, 1966, p. XXX.

7 LOVECCHIO, Antonia, op. cit., p. 106.
} 
politica di Grieco è emblematica del percorso compiuto da tanti, che non senza difficoltà e reticenze si distaccarono da Bordiga per approdare a una nuova concezione del partito e del movimento internazionale» ${ }^{8}$. Dopo aver partecipato al V Congresso del Comintern e, pur tra significative oscillazioni, essersi di nuovo schierato con la sinistra di Bordiga, Grieco è incaricato di ricostituire la Sezione agraria del partito. E' in questo contesto che il comunista foggiano darà forse il meglio di sé, fornendo un contributo fondamentale alla definizione della strategia del PCd'I in riferimento alla questione contadina e, più in generale, allo sviluppo del Mezzogiorno anche dopo la vittoria sul nazifascismo, la nascita del "partito nuovo" e la fondazione della Repubblica.

Nel 1924 Grieco lavora a stretto contatto con Di Vittorio, chiamato a dirigere l'Associazione di difesa dei contadini poveri, fondata dai comunisti fuori dalla Federterra per dare più voce agli ultimi in un difficile contesto politico. La repressione fascista non rappresenta l'unico problema per i comunisti: i rapporti con i socialisti e, in primis, con i riformisti (che sono in netta maggioranza nella CGdL) non favoriscono l'unità interna in quel che rimane delle organizzazioni sindacali nate all'inizio del secolo. Proprio l'analisi di questo complesso contesto è forse poco approfondita dall'autrice e troppo "interna" al mondo comunista. Guardando alla storiografia sui cattolici e sui socialisti, si potevano indagare più a fondo alcuni temi e cesure, tanto più che non viene nascosta da Lovecchio (che pure affronta i rapporti del PCd'I con Miglioli, oltre che con il sardismo e con il Partito dei contadini) la centralità di questi attori nella difficoltà di definire, tra la fine del 1924 e l'inizio del 1925, una piattaforma programmatica efficace da parte della Sezione agraria diretta da Grieco.

Lovecchio, da una parte, evidenzia come in questa fase egli non sfugga alla «identificazione di fascismo e borghesia», interpretazione a sinistra tanto diffusa quanto inadeguata della «natura del fenomeno fascista». Dall'altra, l'autrice sottolinea come il lavoro svolto allora da Grieco - fino alle leggi eccezionali - «fu precipuamente volto alla messa in valore della componente contadina nel quadro della sua alleanza col proletariato, secondo quanto teorizzato in primo luogo da Gramsci»9. Nel giro di qualche mese, Grieco si avvicina definitivamente al nuovo gruppo dirigente. Torna in URSS e, oltre che alla II Conferenza del Krestintern, partecipa alla quinta sessione del Comitato esecutivo allargato del Comintern, quasi un nuovo congresso in cui si discute della "bolscevizzazione" dei partiti comunisti e della "stabilizzazione" del capitalismo. Il contributo di Grieco alla discussione, per Lovecchio, è centrale in merito al rapporto da costruire con le masse contadine. E' l'anticamera delle Tesi agrarie redatte in vista del

\footnotetext{
8 Ibidem, p. 117.

9 Ibidem, pp. 139-140. 
III Congresso del PCd'I, tenutosi clandestinamente a Lione nel gennaio 1926. Qui Grieco, rientrato negli organi dirigenti del partito, si stacca per sempre dalla sinistra di Bordiga. Ma la clandestinità si avvicina, visto che il fascismo sta vincendo e il tardivo ripensamento della linea da parte del Comintern, ora condiviso da Grieco, non può portare a risultati apprezzabili. Per Lovecchio, prima di entrare in clandestinità ed emigrare, il comunista foggiano contribuisce (senza successo) ad «altri due tentativi condotti per intercettare forze politiche non comuniste ma ostili al regime esistenti nel mondo rurale [...]: l'avvicinamento delle masse giovanili cattoliche e l'organizzazione della prima conferenza contadina meridionale» ${ }^{10}$. Con Grieco e Di Vittorio esuli a Parigi, la pratica avrebbe inevitabilmente lasciato spazio alla teoria. Dopo le leggi eccezionali, ricorderà Giorgio Amendola, trovandosi a Mosca Togliatti e Longo, Grieco avrebbe sopportato un enorme carico di lavoro, rappresentando l'unico «elemento permanente di continuità del gruppo dirigente» ${ }^{11}$.

${ }^{10}$ Ibidem, p. 185.

${ }^{11}$ Lovecchio, qui, fa nuovamente riferimento alla prefazione di Giorgio Amendola a GRIECO, op. cit., p. XXXV. 


\section{* L'autore}

Andrea Ricciardi collabora dal 2000 con l'Università degli studi di Milano, dove si è laureato, ha conseguito il dottorato di ricerca in Storia della società e delle istituzioni nell'Europa contemporanea e ha lavorato per quattro anni come assegnista. Si occupa di storia politica e culturale, con particolare riferimento alla storia del movimento operaio e dell'antifascismo. Dal 2010 è membro della redazione de «Il Mestiere di Storico» e dal 2011 del comitato di direzione degli «Annali della Fondazione La Malfa». Oltre a numerosi saggi pubblicati in riviste di settore e volumi collettanei, ha scritto e curato libri su Leo Valiani, Gino Giugni, Vittorio Foa e Riccardo Lombardi. Nel 2013 ha ottenuto l'ASN per professore associato di Storia Contemporanea (11/A3).

URL: < http://www.studistorici.com/progett/autori/\#Ricciardi >

\section{Per citare questo articolo:}

RICCIARDI, Andrea, «Recensione: Antonia LOVECCHIO, Professione rivoluzionario. Per una biografia di Ruggero Grieco (1893-1926), Bari, Edizioni del Sud, 2013, 212 pp.», Diacronie. Studi di Storia Contemporanea : La voce del silenzio: intelligence, spionaggio e conflitto nel XX secolo, 29/12/2016,

URL:<http://www.studistorici.com/2016/12/29/ricciardi_numero_28/ >

\section{Diacronie Studi di Storia Contemporanea $\beta$ www.diacronie.it}

Risorsa digitale indipendente a carattere storiografico. Uscita trimestrale. redazione.diacronie@hotmail.it

Comitato di redazione: Jacopo Bassi - Luca Bufarale - Antonio César Moreno Cantano - Deborah Paci - Fausto Pietrancosta - Alessandro Salvador - Matteo Tomasoni - Luca Zuccolo

Diritti: gli articoli di Diacronie. Studi di Storia Contemporanea sono pubblicati sotto licenza Creative Commons 3.0. Possono essere riprodotti e modificati a patto di indicare eventuali modifiche dei contenuti, di riconoscere la paternità dell'opera e di condividerla allo stesso modo. La citazione di estratti è comunque sempre autorizzata, nei limiti previsti dalla legge. 